\title{
Workflow and Human-Centered Risk Analysis for Novel Mechatronic Rescue Aids
}

\begin{abstract}
Paramedics face rising numbers of deployments every year. As obstacles like stairs occur often, paramedics must frequently manually carry patients and are thereby exposed to loads multitudes higher than recommended. This creates the need for patient transport aids (PTA), which can physically support paramedics in a wide variety of transport situations, without slowing down the transport. In this paper a workflow analysis for transport missions in an urban context and basic tasks for PTAs are presented. Subsequently, the high-level task modelling and human-centered risk analysis according to the HiFEM method are presented for the use case of a patient transport over stairs with a passive PTA, like a rescue chair, and an active PTA like the novel SEBARES prototype. The analysis shows that conventional PTA's have a simple linear use process, however, impose excessive physical workloads, which cause risks like the paramedic or the PTA falling down the stairs. Contrary, active PTA's reduce physical workloads, however, introduce additional concurrent steps, like identifying and correcting misalignments, which create further risks. In order to mitigate risks with active, stair climbing PTAs, either new kinematic designs or intelligent assistance functions, like automatic stair detection, are necessary.
\end{abstract}

Keywords: Patient Transport Aids, Human-Machine Cooperation, Human Risk Analysis, Workflow Modelling, HiFEM

https://doi.org/10.1515/cdbme-2020-3157

\section{Introduction}

Every year over 16 million emergency and non-emergency

\footnotetext{
${ }^{*}$ Corresponding author: Sergey Drobinsky: Chair of Medical Engineering at Helmholtz-Institute for Biomedical Engineering of the RWTH Aachen University, Pauwelsstraße 20, 52074 Aachen, Germany, E-mail: drobinsky@hia.rwth-aachen.de Mark Verjans, Philipp Schleer, Benedikt Kolk, Henrike Bensiek, Klaus Radermacher, Armin Janß: Chair of Medical Engineering at Helmholtz-Institute for Biomedical Engineering of the RWTH Aachen University, Aachen, Germany
}

rescue missions are carried out in Germany and numbers are rising [1]. Previous studies showed that in over $60 \%$ of all patient transports stairs and other obstacles occur, which can lead to the necessity to manually carry patients, who cannot walk on their own [2]. Thereby, due to the demographic change and the fact that over $50 \%$ of German adults are overweight, paramedics often carry loads that significantly exceed recommended limits $[3,4]$. This leads to high rates of occupational accidents and the highest rate of early retirements among all industries [5-7]. In future, this problem will become worse due to increasing numbers of deployments and more female paramedics, who suffer occupational injuries more often $[8,9]$.

Current patient transport aids (PTA) are differentiated in passive and active systems, depending on the level of support they offer: Passive PTAs, like main stretchers, stretcher chairs or spine boards secure patients in a safe position, while all energy for the transportation has to be provided by one or multiple paramedics themselves. Active PTAs offer motorized support and can partially reduce the physical workload, like hydraulic stretchers during lifting or stair climbing rescue chairs during stair transports. However, active aids for stair transports are rarely used by emergency medical services, as they are generally too slow or can only be used for limited use cases. Caterpillar chairs, like the Powertraxx (Ferno, Germany), cannot be used on narrow spiral staircases and may damage stairs as the weight is distributed over the stair edges, while single stair climbers need to be aligned on every single stair.

In the scope of this work the workflow of patient transports with PTAs is analysed and basic tasks for PTAs are identified. Subsequently, a high-level task modelling and risk analysis for the use case of transporting patients over stairs with a passive PTA, like the rescue chair, and an active PTA like the SEBARES system [10] is performed according to the HiFEM method. Finally, the need for automation and human-machine cooperation in the context of patient transports is discussed. 


\section{Materials and Methods}

First, the workflow of urban rescue missions with a PTA is analysed based on expert knowledge of a paramedic with 5 years of experience. All steps directly related to patient transport are considered without regard for concurrent medical treatments that might be performed by paramedics. Subsequently, basic tasks of a PTA are identified that are central to the patient transport.

Subsequently a high-level task modelling is performed for the use case of transporting a patient over stairs with passive and active PTAs and human-centered risks are identified according to the HiFEM method [11]. The use case of performing a stair transport is chosen, as studies showed that it occurs frequently and causes harmful postural workloads [2].

Passive PTAs, like the rescue chair or the main stretcher, provide no motorized support during patient transport, rather all necessary transportation energy is provided by the paramedic. Active PTAs are defined as mechatronic rescue aids that provide external energy for physical support during the entire or parts of the patient transport. Currently, active PTAs can be differentiated in caterpillar systems like the EZ Glider (Ferno, Germany) and single stair climbers like the Scalacombi (Alber, Germany), S-Max (AAT, Germany) or the SEBARES system [10]. Dissipative PTA's, like braked caterpillar chairs, form a special case as they act like active PTA's during downward transport and as passive PTA's during upward transport.

\section{Results}

\subsection{Workflow Analysis}

Rescue missions are conducted in five stages as shown in figure 1: transportation request, arrival at patient site, patient transport preparation, patient transport, and restoring the operational state.

Incoming transportation requests can be either immediate or scheduled depending on whether an emergency or non-emergency transport is required. While in emergency cases transport conditions are often unknown, in scheduled cases the use of ambulances with special equipment, e.g. for obese patients, can be planned. On arrival at the patient's location at least one paramedic approaches the patient immediately, to provide emergency medical care if necessary and already prepare the patient for transportation. Meanwhile, a second paramedic gets the appropriate rescue aid, which depends on various factors like patient characteristics (weight, size), patient's health state (can the patient walk/sit) and environmental factors (does an elevator exist). During transport preparation, all obstacles along the transport route are removed if possible and the patient is transferred on the PTA. Next, the patient is first transported on the PTA to the ambulance, then with the ambulance to the destination address and finally with the PTA to the destination location. During this, it may be necessary to transport the patient over difficult terrain (sloped, rough, soft, slippery) or obstacles (curbs, stairs, doors). Additionally, it may become necessary to relocate the patient multiple times either due to medical reasons, like cramps, or due to transport related reasons, like for safe transportation inside an ambulance. Therefore, it is also necessary to be able to fixate the PTA and the patient respectively in a safe defined position at any point during the entire patient transport, for example during stair transport. After patient transport is completed, the operational state is restored for future patient transports as all equipment including the rescue aid is cleaned, examined and, if necessary, maintained.

Based on the workflow analysis several basic tasks for patient transport with a PTA are identified: maintain a defined safe position when unattended, move with/without patient over different types of terrain and obstacles, facilitate patient relocation and enter/exit ambulance.

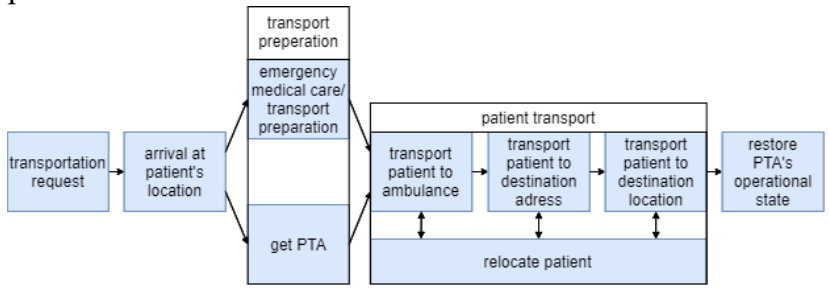

Figure 1: Generalized workflow of a rescue mission with a PTA

\subsection{Human-Centered Risk Analysis}

During patient transport paramedics perform many concurrent tasks besides operating the rescue aid, including but not limited to communicating with patients, relatives and colleagues and monitoring the patient state. Patient transport over stairs can be subdivided in three steps: identifying, planning and performing stair transport. In the following, a general use process is presented for performing a stair transport with passive PTAs in figure 2 and for active PTAs in Figure 3.

For passive PTA's the use process is linear and consists mainly of subsequent action steps. The action steps lift transport aid and carry aid over stairs impose high physical 
workloads on paramedics. As some passive PTAs do not need to be lifted, like the evacuation sheet (Järven Healthcare, Sweden) which is slided down the stairs, the step is marked as optional by brackets.

For active PTA's the use process, compared to the passive case, is more convoluted, the number of tasks is higher, and several instances exist, where perception, cognition and action tasks are performed concurrently. Action steps do not impose high physical workloads except when transportability is not ensured and the PTA must be manually carried, like for example caterpillar chairs in narrow curves. Single stair climbers require high precision during the step align aid before stairs, constant verification of the current alignment while moving over stairs and, when necessary, correction of misalignments. A more detailed high-level task model for the SEBARES system showed that the tasks presented in Figure 3 can be further broken down to create a system- and user-specific task model with basic-level subtasks e.g. pressing a button or verifying the current machine state (including perception, cognition and motoric actions).

During patient transport with a passive PTA excessive physical workloads cause risks, which can have immediate as well as long-term consequences. Failures related to shortterm physical overloads, like the paramedic stumbling or letting go of the PTA, can cause severe immediate effects like injuries to patient and paramedic. Failures related to suboptimal lifting technique, bad posture and repeated unphysiological strains, can lead to musculoskeletal diseases

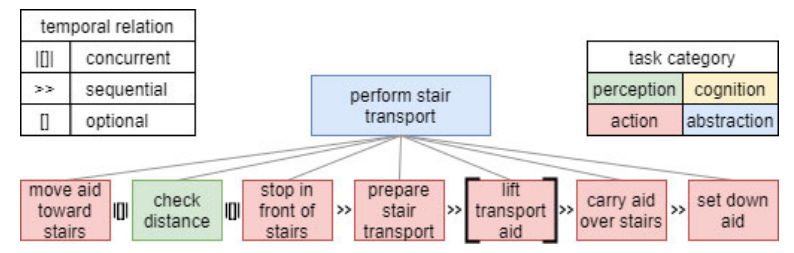

Figure 2: High-level task model for performing a stair transport with a passive PTA

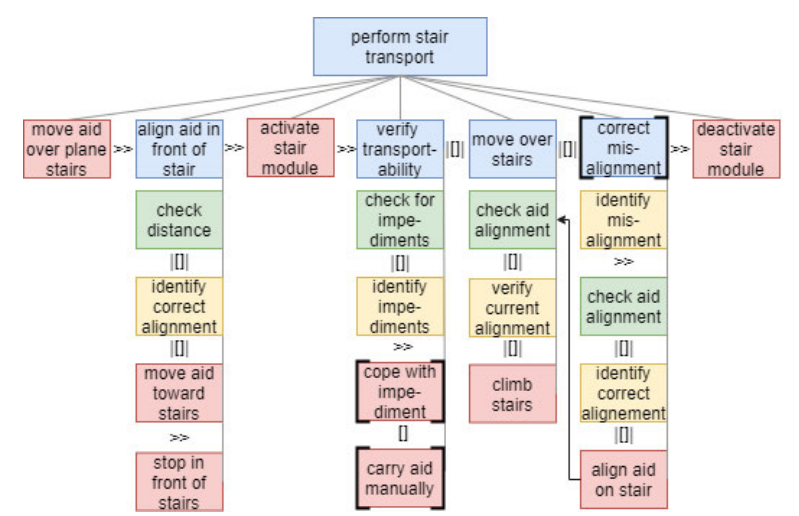

Figure 3: High-level task model for performing a stair transport with an active PTA in the long-term. Both, short- and long-term effects, imply high personal costs (loss in quality of life) and social costs (sick leaves and early retirement). Verjans et al. [2] show that unphysiological strains occur frequently with passive PTAs.

Active PTAs provide external energy to reduce physical loads for paramedics, however, introduce additional tasks. Thereby, main risks arise when transportability cannot be ensured or when PTA misalignments occur. Lack of transportability can be caused by the system specific kinematic design, as is the case with caterpillar PTAs in narrow curves. In these cases, the PTA must be manually carried with all the risks that occur with passive PTAs, however with additional risks related to the stair climbing mechanism, like higher PTA weight and larger size. PTA misalignments can occur due to problems perceiving the alignment, omission of control steps or cognitive overload due to distractions, e.g. during emergency situations. With single stair climbers misalignments can have the severe effect of the PTA falling down steps and injuring the patient and the paramedic downstairs of the PTA. Additionally, in attempts to catch the PTA during the fall, paramedics are exposed to high dynamic loads which, due to the acceleration, exceed the static weight force and can lead to additional injuries. With caterpillar PTAs misalignments can lead to the PTA driving into the wall, which can create the need to manually lift the PTA to correct the misalignment.

\section{Discussion}

Paramedics focus is to transport patients safely and quickly to their destination and ensure the patients well-being in the meantime. However, rising numbers of deployments and high physical workloads lead to a high number of occupational injuries and high rates of early retirement. A study by the institute for occupational safety IFA concludes that transport aids that physically support the paramedics during patient transports, especially during stair transport, are needed [12].

The high-level task model for stair transport with a passive PTA showed that the use process consists of few tasks, which are performed subsequently. The lack of complexity makes it easier to design safe user interaction with the system, however, risks for patient's and paramedics safety arise due to excessive physical workloads. In order to reduce physical workloads, active PTAs provide external energy, however, also introduce additional perception, cognition and action tasks, which lead to a more convoluted use process, with many concurrent steps. Thereby, the analysis showed that two central risks for current active PTAs arise: PTA misalignment, which can lead to the PTA 
falling down the stairs, and not ensured transportability, which can lead to the necessity to manually carry the PTA. The two current types of active PTA's, single stair climbers and caterpillar climbers, show a trade-off: Single stair climbers are prone to misalignments with severe effects like injuring the patient and the paramedics. Caterpillar climbers, however, are limited in their applicability due to their long inflexible stair module, that cannot be used in narrow curves.

Considering that, besides operating the PTA, paramedics perform several concurrent tasks, like monitoring the patient's state or communicating with family members, high cognitive loads during patient transport and especially in emergency situations can occur. The high number of concurrent tasks that need to be performed to verify alignment and transportability during stair transport with active PTAs can introduce additional cognitive loads and lead to cognitive overload. This in turn can lead to errors, like failing to recognize misalignments. Therefore, it is necessary to limit the consequences of misalignments and their occurrence as well as to help the paramedic to detect them in time, while maintaining transportability for all occurring transport conditions.

Solutions may either be new kinematic designs for stair climbers or intelligent assistance systems. The latter was shown to be a viable option to reduce mental workloads in various domains, like assisted driving and robotic surgery $[13,14]$. Thereby, assistance systems can be provided with different levels of automation. In the analysed use case of transporting a patient over stairs, assistance systems could either provide an automatic recognition and correction of misalignments or only a warning and a proposal of counter measures. However, when designing assistance systems a human- and use-centered risk analysis for the specific system is essential in order to identify, which functions need to be automated and to what degree, because badly designed automation can lead, among others, to mode confusion or overreliance [15]. Therefore, further research is necessary to either find kinematic solutions or develop assistance systems for flexible and robust stair climbing with active PTA's. However, when developing the latter, it may be beneficial to re-evaluate the relationship between paramedic and PTA from human-machine interaction to human-machine cooperation, as proposed by Hoc [16].

\section{Conclusion}

In order to decrease the burden on paramedics, active PTAs need to enable effective, efficient and safe patient transport for all common transport scenarios. Therefore, either new kinematic designs or intelligent assistance functions like obstacle recognition or automated alignment verification need to be developed for robust and flexible stair climbing.

\section{References}

[1] Schmiedel R. Leistungen des Rettungsdienstes 2016/17. Bremen: Fachverlag NW in der Carl Ed. Schünemann KG 2019.

[2] Verjans M, Schütt A, Schleer P, Struck D, Radermacher K. Postural workloads on paramedics during patient transport. Current Directions in Biomedical Engineering 2018; 4(1): 161-4

[3] Statistisches Bundesamt. Pressemitteilung Nr. 14 vom 2. April 2019 [cited 09.03.20] Available from: URL: https://www.destatis.de/DE/Presse/Pressemitteilungen/Zahlder-Woche/2019/PD19_14_p002.html.

[4] Hettinger T. Gewichtsgrenzen für das höchstzulässige Heben und Tragen von Lasten durch männliche und weibliche sowie jugendliche Arbeitnehmer 1981.

[5] Maguire BJ, Hunting KL, Guidotti TL, Smith GS. Occupational injuries among emergency medical services personnel. Prehosp Emerg Care 2005; 9(4): 405-11

[6] Rodgers LM. A five year study comparing early retirements on medical grounds in ambulance personnel with those in other groups of health service staff. Part II: Causes of retirements. Occup Med (Lond) 1998; 48(2): 119-32

[7] Pattani S, Constantinovici N, Williams S. Who retires early from the NHS because of ill health and what does it cost? A national cross sectional study. BMJ 2001; 322(7280): 208-9

[8] Maguire BJ, Smith S. Injuries and fatalities among emergency medical technicians and paramedics in the United States. Prehosp Disaster Med 2013; 28(4): 376-82

[9] Statistisches Bundesamt. Gesundheitspersonal (Vollzeitäquivalente vorläufig) in 1.000. [cited 09.03.20] Available from: URL: http://www.gbe-bund.de.

[10] Verjans M, Phlippen L, Schleer P, Radermacher K. SEBARES - Design and Evaluation of a Controller for a novel externally guided self-balancing patient rescue aid. In: 2019 18th European Control Conference; 2019; 209-14.

[11] Janß A, Lauer W, Radermacher K. Bewertung sicherheitskritischer Systeme im OperationssaalEvaluation of Risk-Sensitive Systems in the OR 2009; 8(1): 257

[12] [Schiefer C, Hermanns I, Schuster D, Brand K, Ditchen D. Untersuchung der physischen Belastungen von Rettungskräften beim Patiententransport in Treppenhäusern: Institut für Arbeitsschutz; 08.2019.

[13] Takada Y, Shimoyama O. Evaluation of Driving-Assistance Systems Based on Drivers' Workload. In: Evaluation of Driving-Assistance Systems Based on Drivers' Workload; 2001. lowa City, lowa: University of lowa; 208-13.

[14] Schleer P, Drobinsky S, Radermacher K. Evaluation of Different Modes of Haptic Guidance for Robotic Surgery. IFAC-PapersOnLine 2019; 51(34): 97-103

[15] O'Malley MK. Principles of human-machine interfaces and interactions 2007.

[16] Hoc J-M. Human-Machine Cooperation. In: The Oxford Handbook of Cognitive Engineering 2013. 